BA-03-21

FERMILAB-Pub-03/405-T

hep-ph/0312224

December 2003

\title{
Leptogenesis in the type III seesaw mechanism
}

\author{
Carl H. Albright \\ Department of Physics, Northern Illinois University, DeKalb, IL 60115 \\ and \\ Fermi National Accelerator Laboratory, P.O. Box 500, Batavia, IL 60510* \\ S.M. Barr \\ Bartol Research Institute, University of Delaware, Newark, DE $19716^{\dagger}$
}

\begin{abstract}
It is shown that the type III seesaw mechanism proposed recently can have certain advantages over the conventional (or type I) seesaw mechanism for leptogenesis. In particular a resonant enhancement of leptogenesis via heavy quasi-Dirac right-handed neutrino pairs can occur without a special flavor form or "texture" of the mass matrices being assumed. Some of the requirements for neutrino mixing and leptogenesis are effectively decoupled.
\end{abstract}

*electronic address: albright@fnal.gov

†electronic address: smbarr@bartol.udel.edu 


\section{INTRODUCTION}

Recently there has been much interest in the idea of leptogenesis through the decay of heavy right-handed neutrinos [1-4]. In part, this interest is due to the fact that such a scenario links the origin of matter to the masses and mixings of neutrinos, about which we are now learning from experiment. Most analyses of leptogenesis have focussed on the conventional seesaw mechanism for neutrino mass [5], which is sometimes called the "type I seesaw mechanism" to distinguish it from the "type II seesaw mechanism" discussed in [6] involving Higgs triplets. An analysis of thermal leptogenesis in models where the type II mechanism operates has been given in Ref. [7].

In a recent paper [8] it was shown that in a wide class of models based on $S O(10)$ (or larger groups) a somewhat different seesaw mechanism can operate that has several interesting features. In particular, this "type III seesaw" may have less difficulty in reproducing realistic neutrino masses and mixings than does the conventional type I seesaw. At least, this was found to be the case in a particular, rather typical, realistic $S O(10)$ model of quark and lepton masses [9]. In the present paper we show that the type III seesaw mechanism also has some significant advantages for leptogenesis.

In realistic SUSY GUT models, it is typically found that for there to be sufficient leptogenesis there must be resonant enhancement [3] coming from two superheavy "right-handed" neutrinos (call them $N_{1}$ and $N_{2}$ ) forming what we shall call a "nearly degenerate pseudoDirac pair". What we mean by this is that they have a mass matrix of the form

$$
\left(N_{1}, N_{2}\right)\left(\begin{array}{cc}
\Delta_{11} & M \\
M & \Delta_{22}
\end{array}\right)\left(\begin{array}{l}
N_{1} \\
N_{2}
\end{array}\right),
$$

with $\Delta_{i i} \ll M$. This is equivalent, upon diagonalization, to two Majorana neutrinos $N_{+} \cong$ $\left(N_{1}+N_{2}\right) / \sqrt{2}$ and $N_{-} \cong\left(N_{1}-N_{2}\right) / \sqrt{2}$, with masses approximately equal to $M+\frac{1}{2}\left(\Delta_{11}+\Delta_{22}\right)$ and $-M+\frac{1}{2}\left(\Delta_{11}+\Delta_{22}\right)$ respectively. Then the lepton asymmetry produced by the decays of these heavy neutrinos will be enhanced by a factor of $I \cong \frac{M}{2\left(\Delta_{11}+\Delta_{22}\right)}$. In many models this factor must be very large to obtain sufficient leptogenesis [10].

In the conventional or type I seesaw, the requirement for resonant enhancement that there be such a nearly degenerate right-handed neutrino pair places a very strong constraint on the form of $M_{R}$, the mass matrix of the right-handed neutrinos. The matrix $M_{R}$ also appears in the type I seesaw formula for the mass matrix of the observed light neutrinos: $M_{\nu}=-M_{N} M_{R}^{-1} M_{N}^{T}$. (Here and henceforth $M_{N}$ denotes the Dirac mass matrix that links the left-handed and right-handed neutrinos.) The leptogenesis constraints on $M_{R}$ can therefore clash with what is needed to give a realistic pattern of light neutrino masses and mixings. Indeed, in Ref. [11] it was found that this does happen in a typical realistic $S O(10)$ model of quark and lepton masses.

In the type III seesaw, the right-handed neutrino mass matrix does not enter in the same way into the formula for $M_{\nu}$ as in the type I seesaw. Consequently, the issues of leptogenesis and of realistic light neutrino masses and mixings are not so tightly coupled, and the clash referred to above can easily be avoided. Moreover, nearly degenerate pseudo-Dirac pairs of heavy neutrinos arise very simply and naturally in the type III seesaw mechanism, as will be seen. Their existence does not impose any special form in flavor space on the mass 
matrices involved. In fact, in the type III seesaw mechanism there are not (as in type I) three superheavy Majorana neutrinos, but rather six superheavy Majorana neutrinos, which form three pseudo-Dirac pairs. One or more of these pairs can be highly degenerate without any special "textures" or forms.

\section{TYPE III SEESAW MECHANISM}

In this section we shall briefly review the type III seesaw mechanism. More details can be found in Ref. [8]. The type III seesaw mechanism arises in $S O(10)$ models in which the breaking of $B-L$ is done by spinor $\mathbf{1 6}_{H}+\overline{\mathbf{1 6}}_{H}$ Higgs fields rather than by rank-five tensor $\mathbf{1 2 6}_{H}+\overline{\mathbf{1 2 6}}_{H}$ Higgs fields. (The generalization to larger groups, like $E_{6}$ is straightforward.) In such a case, the right handed neutrino masses must come from an effective $d=5$ operator of the form

$$
O_{e f f}=\lambda_{i j a b}\left(\mathbf{1 6}_{i} \mathbf{1 6}_{j}\right) \overline{\mathbf{1 6}}_{H}^{a} \overline{\mathbf{1 6}}_{H}^{b} / M,
$$

The $\mathbf{1 6}_{i}, i=1,2,3$ are the three families of light quarks and leptons. The indices $a$ and $b$ on the Higgs multiplets indicate that there can be more than one $\overline{\mathbf{1 6}}_{H}$. We will denote by $\mathbf{p}(\mathbf{q})$ an $S U(5) \mathbf{p}$ representation contained in an $S O(10) \mathbf{q}$ representation. Thus, the left-handed neutrinos $\nu_{i}$ are contained in $\overline{\mathbf{5}}\left(\mathbf{1 6}_{i}\right)$, while the left-handed antineutrinos $N_{i}^{c}$ (the conjugates of the right-handed neutrinos) are contained in $\mathbf{1}\left(\mathbf{1 6}_{i}\right)$. The $N_{i}^{c}$ obtain superlarge mass from $O_{\text {eff }}$ when the $\mathbf{1}\left(\overline{\mathbf{1 6}}_{H}^{a}\right)$ obtain GUT-scale vacuum expectation values $(\mathrm{VEVs}):\left\langle\mathbf{1}\left(\overline{\mathbf{1 6}}_{H}^{a}\right)\right\rangle \equiv \Omega_{a}$. Thus $O_{\text {eff }}$ gives a term of the form $N_{i}^{c} N_{j}^{c}\left(\lambda_{i j a b} \Omega_{a} \Omega_{b} / M\right)$.

The operator $O_{\text {eff }}$ must arise from integrating out some superheavy states, as shown in Fig. 1. For simplicity, we assume that the states integrated out are $S O(10)$ singlets, though what representations of $S O(10)$ they are in does not really matter for the later discussion. Suppose that there are three such singlets, one for each family, denoted $S_{i} \equiv \mathbf{1}_{i}$. The terms needed in the Yukawa superpotential to give $O_{\text {eff }}$ from the diagram of Fig. 1 are

$$
W_{R H \nu}=F_{i j}^{a}\left(\mathbf{1 6}_{i} \mathbf{1}_{j}\right) \overline{\mathbf{1 6}}_{H}^{a}+\mathcal{M}_{i j} \mathbf{1}_{i} \mathbf{1}_{j} .
$$

There must also be a Dirac neutrino mass term $\left(M_{N}\right)_{i j} \nu_{i} N_{j}^{c}$. In a realistic model $M_{N}$ would typically come from a combination of several operators at the $S O(10)$ level. For our purposes, the origin of $M_{N}$ does not matter.

The crucial point for the type III seesaw mechanism is that the weak-doublet Higgs fields contained in the $\overline{\mathbf{1 6}}_{H}^{a}$ can get weak-scale VEVs: $\mid\left\langle\mathbf{5}\left(\overline{\mathbf{1 6}}_{H}^{a}\right\rangle\right| \equiv u_{a}$. These VEVs directly couple the left-handed neutrinos $\nu_{i}$ to the singlets $S_{i}$. The full mass matrix of neutral leptons has the form:

$$
W_{\text {neut }}=\left(\nu_{i}, N_{i}^{c}, S_{i}\right)\left(\begin{array}{ccc}
0 & \left(M_{N}\right)_{i j} & F_{i j}^{a} u_{a} \\
\left(M_{N}\right)_{j i} & 0 & F_{i j}^{a} \Omega_{a} \\
F_{j i}^{a} u_{a} & F_{j i}^{a} \Omega_{a} & \mathcal{M}_{i j}
\end{array}\right)\left(\begin{array}{c}
\nu_{j} \\
N_{j}^{c} \\
S_{j}
\end{array}\right)
$$

The matrix $\mathcal{M}_{i j}$ and the VEVs $\Omega_{a}$ are superheavy, while the mass matrix $M_{N}$ and the VEVs $u_{a}$ are of order the weak scale. In the special case where there is only one $\overline{\mathbf{1 6}}_{H}$ the situation becomes very simple, for then the $\nu S$ block $\left(=F_{i j} u\right)$ is proportional to the $N^{c} S$ 
block $\left(=F_{i j} \Omega\right)$. Consequently a rotation of $\nu_{i}$ and $N_{i}^{c}$ by an angle $\tan ^{-1}(u / \Omega)$ makes the $\nu S$ and $S \nu$ blocks exactly vanish and induces in the $\nu \nu$ block the entry $-\left(M_{N}+M_{N}^{T}\right) u / \Omega$. The rest of the matrix has the standard "double seesaw" form. The total effective mass matrix for the three light neutrinos (neglecting terms subleading in $M_{W} / M_{G U T}$ ) is

$$
M_{\nu}=-M_{N} M_{R}^{-1} M_{N}^{T}-\left(M_{N}+M_{N}^{T}\right) \frac{u}{\Omega}
$$

where

$$
M_{R}=(F \Omega) \mathcal{M}^{-1}\left(F^{T} \Omega\right)
$$

In the general case where there is more than one $\overline{\mathbf{1 6}}_{H}$, the light neutrino mass matrix has the form

$$
M_{\nu}=-M_{N} M_{R}^{-1} M_{N}^{T}-\left(M_{N} H+H^{T} M_{N}^{T}\right) \frac{u}{\Omega}, \quad H \equiv\left(F^{\prime} F^{-1}\right)^{T},
$$

where $\Omega \equiv\left(\sum_{a} \Omega_{a}^{2}\right)^{1 / 2}, u \equiv\left(\sum_{a} u_{a}^{2}\right)^{1 / 2}, F_{i j} \equiv \sum_{a} F_{i j}^{a} \Omega_{a} / \Omega$, and $F_{i j}^{\prime} \equiv \sum_{a} F_{i j}^{a} u_{a} / u$.

The first term in Eq. (7) is the usual type I seesaw contribution. The second term is the type III seesaw contribution. If the elements of the matrix $\mathcal{M}_{i j}$ are small compared to those of $F_{i j}^{a} \Omega_{a}$, then it is easy to see from Eqs. (6) and (7) that the type I contribution becomes negligible compared to the type III contribution. We shall be interested in this case, which is also favorable for leptogenesis, as will be seen.

In the limit that $\mathcal{M}_{i j}=0$ one sees from Eq. (4) that the superheavy neutrinos have simply the mass term $F_{i j} \Omega\left(N_{i}^{c} S_{j}\right)$. That is, the $N_{i}^{c}$ and $S_{i}$ pair up to form three Dirac neutrinos. On the other hand, if $\mathcal{M}_{i j}$ is small (compared to $F_{i j} \Omega$ ) but not zero, then these three Dirac neutrinos get slightly split into six eigenstates forming three nearly degenerate pseudo-Dirac neutrinos. It is this fact that can be exploited to enhance leptogenesis.

\section{LEPTOGENESIS IN A REALISTIC TYPE III MODEL}

Rather than attempting to study leptogenesis in type III seesaw models in complete generality, it may be more illuminating to study it in a specific realistic model of quark and lepton (including neutrino) masses and mixings. We shall use the $S O(10)$ model of Ref. [9].

One of the attractive features of $S O(10)$ unification (or unification based on larger groups) is that the unified symmetry relates the Dirac mass matrix of the neutrinos, $M_{N}$, to the mass matrices of the up quarks, down quarks and charged leptons. It is therefore possible to construct very predictive models. In a sufficiently predictive model, the quark and charged lepton masses and CKM angles can be enough to determine $M_{N}$ completely. Such is the case in the model of Ref. [9], which is therefore a good laboratory for studying leptogenesis. In that very predictive and realistic model the Dirac neutrino mass matrix is given by

$$
M_{N}=\left(\begin{array}{ccc}
\eta & 0 & 0 \\
0 & 0 & \epsilon \\
0 & -\epsilon & 1
\end{array}\right) v_{u}
$$


where $\eta \cong 0.6 \times 10^{-5}$ and $\epsilon \cong 0.14$. The $S O(10)$ symmetry does not directly relate the matrices $F_{i j}$ and $F_{i j}^{\prime}$ to anything known, and so there is considerable freedom in choosing their forms, and consequently also the form of the matrix $H_{i j}$. As pointed out in Ref. [8], there are two simple cases which lead to realistic patterns of masses and mixings for the light neutrinos $\nu_{i}$.

In Case 1, all the elements of $F_{i j}$ and of $F_{i j}^{\prime}$ are of order $f$, some dimensionless parameter. Then

$$
H_{i j} \sim\left(\begin{array}{lll}
1 & 1 & 1 \\
1 & 1 & 1 \\
1 & 1 & 1
\end{array}\right)
$$

and therefore, by Eq. (7), the type III seesaw mass formula gives

$$
M_{\nu} \sim\left(\begin{array}{ccc}
\eta & \epsilon & 1 \\
\epsilon & \epsilon & 1 \\
1 & 1 & 1
\end{array}\right) \frac{v_{u} u}{\Omega} .
$$

It is evident by inspection of the 12 and 23 elements of $M_{\nu}$ that the solar and atmospheric angles naturally come out of the right order. However, some very mild tuning (if one should even call it that) of order $10^{-1}$ is needed to make the 13 element small enough to satisfy the experimental constraint on $U_{e 3}$. In Case 1 all the right-handed neutrinos have mass of the same order, namely of order $\Omega \sim M_{G U T}$. This is not good for thermal leptogenesis, as it would require a reheating temperature of order $M_{G U T}$, leading to the overproduction of gravitinos.

In Case 2, both $F_{i j}$ and $F_{i j}^{\prime}$ have the form

$$
F, F^{\prime} \sim\left(\begin{array}{ccc}
\lambda^{2} & \lambda & \lambda \\
\lambda & 1 & 1 \\
\lambda & 1 & 1
\end{array}\right),
$$

where $\lambda \sim \eta / \epsilon$. This form can naturally arise from an abelian flavor symmetry under which the first family has a different charge from the other two. One would therefore assume that $\mathcal{M}_{i j}$ also has the form shown in Eq. (11). Such an abelian flavor symmetry would also explain why the element $\eta$ in Eq. (8) is so much smaller than the other non-zero elements in $M_{N}$. From Eqs. (7) and (11),

$$
H \sim\left(\begin{array}{ccc}
1 & \epsilon / \eta & \epsilon / \eta \\
\eta / \epsilon & 1 & 1 \\
\eta / \epsilon & 1 & 1
\end{array}\right)
$$

and

$$
M_{\nu} \sim\left(\begin{array}{ccc}
\eta & \epsilon & \epsilon \\
\epsilon & \epsilon & 1 \\
\epsilon & 1 & 1
\end{array}\right) \frac{v_{u} u}{\Omega} .
$$

Note that this naturally gives all three neutrino mixing angles of the correct order: $\left|U_{\mu 3}\right| \sim 1$, $\left|U_{e 2}\right| \sim 1$, and $\left|U_{e 3}\right| \sim \epsilon$. 
In the limit where the matrix $\mathcal{M}_{i j}$ can be neglected, the superheavy neutrino masses are given simply by the matrix $F_{i j} \Omega$. Therefore, in Case 2, two of the superheavy (Dirac) neutrinos have mass of order $\Omega \sim M_{G U T}$ and one superheavy (Dirac) neutrino has mass of order $\lambda^{2} \Omega \sim 10^{7} \mathrm{GeV}$, which is quite satisfactory from the point of view of thermal leptogenesis. This is the case that we shall now consider in more detail.

To analyze leptogenesis it is convenient to go to a basis in which the matrix $F_{i j}$ is diagonal. We shall indicate quantities in this basis with a tilde. To reach this basis one does unitary transformations $N_{i}^{c}=U_{i j} \tilde{N}_{j}^{c}$ and $S_{i}=V_{i j} \tilde{S}_{j}$. From the assumed form of $F_{i j}$ in Eq. (11) we can write the matrix $U_{i j}$ as

$$
U=\left(\begin{array}{ccc}
u_{11} & \lambda u_{12} & \lambda u_{13} \\
\lambda u_{21} & u_{22} & u_{23} \\
\lambda u_{31} & u_{32} & u_{33}
\end{array}\right)
$$

where $u_{i j} \sim 1$, and by unitarity $u_{11} \cong 1$. The unitary matrix $V_{i j}$ has a similar form; however, we shall not need to parametrize it. The elements of the diagonalized matrix $\tilde{F}_{i j} \Omega=\left(U_{k i} F_{k \ell} V_{\ell j}\right) \Omega$ can be written

$$
\tilde{F} \Omega=\left(\begin{array}{ccc}
\lambda^{2} F_{1} & 0 & 0 \\
0 & F_{2} & 0 \\
0 & 0 & F_{3}
\end{array}\right) \Omega \equiv\left(\begin{array}{ccc}
M_{1} & 0 & 0 \\
0 & M_{2} & 0 \\
0 & 0 & M_{3}
\end{array}\right),
$$

where $F_{i} \sim 1$. In the new basis the matrices $\tilde{F}_{i j}^{\prime} u$ and $\tilde{\mathcal{M}}_{i j}$ are not diagonal, but rather retain the same basic form shown in Eq. (11), so that they can be parametrized as follows:

$$
\tilde{F}^{\prime} u=\left(\begin{array}{ccc}
\lambda^{2} f_{11} & \lambda f_{12} & \lambda f_{13} \\
\lambda f_{21} & f_{22} & f_{23} \\
\lambda f_{31} & f_{32} & f_{33}
\end{array}\right) v_{u}, \quad \tilde{\mathcal{M}}=\left(\begin{array}{ccc}
\lambda^{2} g_{11} & \lambda g_{12} & \lambda g_{13} \\
\lambda g_{21} & g_{22} & g_{23} \\
\lambda g_{31} & g_{32} & g_{33}
\end{array}\right) M_{S},
$$

where $f_{i j}, g_{i j} \sim 1$. The magnitude of $M_{S}$ is assumed to be much less than $\Omega$. As will be seen, this is necessary for sufficient leptogenesis.

The Dirac neutrino mass matrix $M_{N}$ goes to $\tilde{M}_{N}=M_{N} U$, so that from Eqs. (8) and (14) it is given by

$$
\tilde{M}_{N} \cong\left(\begin{array}{ccc}
\eta u_{11} & \eta \lambda u_{12} & \eta \lambda u_{13} \\
\epsilon \lambda u_{31} & \epsilon u_{32} & \epsilon u_{33} \\
\lambda u_{31} & u_{32} & u_{33}
\end{array}\right) v_{u} \equiv \tilde{Y} v_{u}
$$

The type III seesaw contribution to the light neutrino mass matrix (which dominates, since $M_{S} \ll \Omega$ ) is determined from Eq. (7) to be

$$
M_{\nu} \cong-\left[\begin{array}{ccc}
2 \eta\left(\frac{u_{11} f_{11}}{F_{1}}\right) & \frac{\eta}{\lambda}\left(\frac{u_{11} f_{21}}{F_{1}}\right) & \frac{\eta}{\lambda}\left(\frac{u_{11} f_{31}}{F_{1}}\right) \\
\frac{\eta}{\lambda}\left(\frac{u_{11} f_{21}}{F_{1}}\right) & 2 \epsilon \sum_{j}\left(\frac{u_{3 j} f_{2 j}}{F_{j}}\right) & \sum_{j}\left(\frac{u_{3 j} f_{2 j}}{F_{j}}\right) \\
\frac{\eta}{\lambda}\left(\frac{u_{11} f_{31}}{F_{1}}\right) & \sum_{j}\left(\frac{u_{33} f_{2 j}}{F_{j}}\right) & 2 \sum_{j}\left(\frac{u_{3 j} f_{3 j}}{F_{j}}\right)
\end{array}\right]\left(\frac{v_{u}^{2}}{\Omega}\right) .
$$

Since $\lambda$ is presumed to be of order $\eta / \epsilon$, one sees that $M_{\nu}$ does indeed have the form given in Eq. (13). 
The six superheavy two-component neutrinos have the mass matrix

$$
\left(\tilde{N}_{i}^{c}, \tilde{S}_{i}\right)\left(\begin{array}{cc}
0 & M_{i} \delta_{i j} \\
M_{i} \delta_{i j} & \tilde{\mathcal{M}}_{i j}
\end{array}\right)\left(\begin{array}{c}
\tilde{N}_{j}^{c} \\
\tilde{S}_{j}
\end{array}\right)
$$

where $\tilde{\mathcal{M}}_{i j}$ is given in Eq. (16). Leptogenesis is almost exclusively produced by the decays of the lightest pair of these superheavy neutrinos, which have effectively the two-by-two mass matrix

$$
\left(\tilde{N}_{1}^{c}, \tilde{S}_{1}\right)\left(\begin{array}{cc}
0 & M_{1} \\
M_{1} & \tilde{\mathcal{M}}_{11}
\end{array}\right)\left(\begin{array}{c}
\tilde{N}_{1}^{c} \\
\tilde{S}_{1}
\end{array}\right)=\left(\tilde{N}_{1}^{c}, \tilde{S}_{1}\right) \lambda^{2}\left(\begin{array}{cc}
0 & F_{1} \Omega \\
F_{1} \Omega & g_{11} M_{S}
\end{array}\right)\left(\begin{array}{c}
\tilde{N}_{1}^{c} \\
\tilde{S}_{1}
\end{array}\right)
$$

If, as we assume, $M_{S} \ll \Omega$, these form an almost degenerate pseudo-Dirac pair, or equivalently two Majorana neutrinos with nearly equal and opposite masses. These Majorana neutrinos are $N_{ \pm} \cong\left(\tilde{N}_{1}^{c} \pm \tilde{S}_{1}\right) / \sqrt{2}$, with masses $M_{ \pm} \cong \pm M_{1}+\frac{1}{2} \tilde{\mathcal{M}}_{11}=\lambda^{2}\left( \pm F_{1} \Omega+\frac{1}{2} g_{11} M_{S}\right)$. These can decay into light neutrino plus Higgs via the term $Y_{i \pm}\left(N_{ \pm} \nu_{i}\right) H$, where

$$
Y_{i \pm} \cong\left(\tilde{Y}_{i 1} \pm \tilde{F}_{i 1}^{\prime}\right) / \sqrt{2} \mp \frac{\tilde{\mathcal{M}}_{11}}{4 M_{1}}\left(\tilde{Y}_{i 1} \mp \tilde{F}_{i 1}^{\prime}\right) / \sqrt{2}
$$

Here $\tilde{Y}$ is the Dirac Yukawa coupling matrix given in Eq. (17). It is straightforward to show that the lepton asymmetry per decay produced by the decays of $N_{ \pm}$is given (in the now usual notation) by [2-4]

$$
\epsilon_{1}=\frac{1}{4 \pi} \frac{\operatorname{Im}\left[\sum_{j}\left(Y_{j+} Y_{j-}^{*}\right)\right]^{2}}{\sum_{j}\left[\left|Y_{j+}\right|^{2}+\left|Y_{j-}\right|^{2}\right]} I\left(M_{-}^{2} / M_{+}^{2}\right)
$$

where $I\left(M_{-}^{2} / M_{+}^{2}\right)$ comes from the absorptive part of the decay amplitude of $N_{ \pm}$. This function is given by $I(x)=\sqrt{x}\left[1-(1+x) \ln \left(1+\frac{1}{x}\right)+\frac{1}{1-x}\right]$. Thus $I\left(M_{-}^{2} / M_{+}^{2}\right) \cong M_{1} / 2 \tilde{\mathcal{M}}_{11}=$ $\left(F_{1} / 2 g_{11}\right)\left(\Omega / M_{S}\right)$. We will call this the resonant enhancement factor. It can be large if $M_{S} \ll$ $\Omega$. The expression for $I\left(M_{-}^{2} / M_{+}^{2}\right)$ given above is only valid when the mass splitting $\left|M_{+}\right|-$ $\left|M_{-}\right|=\tilde{\mathcal{M}}_{11}$ is larger than the widths of the $N_{ \pm}$, which are given by $\Gamma_{ \pm} \cong \frac{1}{8 \pi} M_{1} \sum_{k}\left|Y_{k \pm}\right|^{2}$. From Eqs. (16), (17), and (21), one sees that $\Gamma_{ \pm} \sim \lambda^{2} M_{1} / 8 \pi$. Thus the condition for maximum lepton asymmetry resulting from resonant enhancement, that the splitting of $N_{ \pm}$ be comparable to their widths [12], only constrains the enhancement factor to be less than about $8 \pi / \lambda^{2} \sim 10^{10}$. This is not a problem as we shall only need enhancements of order $10^{5}$ or so.

Making use of Eqs. (21) and (22) one obtains

$$
\epsilon_{1}=\frac{1}{4 \pi} \frac{\sum_{j}\left(\left|\tilde{Y}_{j 1}\right|^{2}-\left|\tilde{F}_{j 1}^{\prime}\right|^{2}\right) \operatorname{Im}\left(\sum_{k} \tilde{Y}_{k 1}^{*} \tilde{F}_{k 1}^{\prime}\right)}{\sum_{j}\left(\left|\tilde{Y}_{j 1}\right|^{2}+\left|\tilde{F}_{j 1}^{\prime}\right|^{2}\right)} I\left(M_{-}^{2} / M_{+}^{2}\right),
$$

This can be evaluated in terms of the parameters of the model using Eqs. (16) and (17), giving

$$
\epsilon_{1} \cong \frac{\lambda^{2}}{4 \pi}\left[\frac{\left(\left|u_{31}\right|^{2}-\left|f_{31}\right|^{2}\right) \operatorname{Im}\left(u_{31}^{*} f_{31}\right)}{\left|u_{31}\right|^{2}+\left|f_{31}\right|^{2}+\left|f_{21}\right|^{2}}\right] I
$$


The lepton asymmetry of the universe is given by [2-4]

$$
Y_{L}=\epsilon_{1} d / g_{*},
$$

where $d$ is the washout parameter and $g_{*}$ is the effective number of polarization states at the time of the $N_{ \pm}$decays. In a supersymmetric model $g_{*}=228.75$. The washout parameter is given approximately by [13]

$$
d=\frac{0.3}{k(\ln k)^{3 / 5}}, \quad k \equiv \frac{\tilde{m}_{1}}{10^{-3} \mathrm{eV}},
$$

where

$$
\tilde{m}_{1} \equiv \frac{8 \pi v_{u}^{2} \Gamma_{N_{ \pm}}}{M_{N_{ \pm}}^{2}} \cong \lambda^{2} \frac{v_{u}^{2}}{M_{1}}\left(\left|u_{31}\right|^{2}+\left|f_{31}\right|^{2}+\left|f_{21}\right|^{2}\right) .
$$

Before further analyzing leptogenesis in this model, it is useful to find the constraints imposed on its parameters by the condition that realistic masses and mixings arise for the light neutrinos. The two conditions that are important are that $m_{3}$ (the largest of the light neutrino masses) and $\theta_{12}$ (the solar neutrino mixing angle) come out right. One expects that $m_{3} \simeq\left(M_{\nu}\right)_{33}=2 \sum_{j}\left(u_{3 j} f_{3 j} / F_{j}\right)\left(v_{u}^{2} / \Omega\right)$, cf. Eq. (18). It will turn out to be convenient to express this in terms of the following parameter:

$$
a \equiv \sum_{j}\left[\frac{u_{3 j} f_{3 j}}{\left|f_{21}\right|^{2}} \frac{F_{1}}{F_{j}}\right]
$$

which is naturally of order one. Then one requires that

$$
m_{3} \simeq \frac{2\left|f_{21}\right|^{2}}{F_{1}} a \frac{v_{u}^{2}}{\Omega} \simeq 0.06 \mathrm{eV} .
$$

Since $2 v_{u}^{2} / m_{3} \simeq 10^{15} \mathrm{GeV}$,

$$
\Omega \simeq \frac{\left|f_{21}\right|^{2} a}{F_{1}}\left[10^{15} \mathrm{GeV}\right] .
$$

Using the fact that $M_{1}=\lambda^{2} F_{1} \Omega$ (cf. Eq. (15)), this can also be written in the following form, which shall be useful shortly:

$$
\frac{\lambda^{2}}{M_{1}} \simeq \frac{1}{\left|f_{21}\right|^{2} a}\left(10^{15} \mathrm{GeV}\right)^{-1} .
$$

The condition that the solar angle satisfy $\tan ^{2} \theta_{12} \simeq 0.4$ implies that $\left(M_{\nu}\right)_{12} \simeq\left(M_{\nu}\right)_{22}$. From the fact that $m_{2} \simeq m_{3} / 6$ (if the light neutrino masses have an ordinary hierarchy), it follows that $\left(M_{\nu}\right)_{12} \simeq 0.1 m_{3}$ and hence, from Eq. (18), that $(\eta / \lambda)\left(\left|f_{21}\right| / F_{1}\right)\left(v_{u}^{2} / \Omega\right) \simeq$ $0.006 \mathrm{eV}$. Again using the formula for $M_{1}$ and the value of $\eta$, one arrives at the result that

$$
\frac{\lambda}{M_{1}} \simeq \frac{1}{\left|f_{21}\right|}\left(3 \times 10^{10} \mathrm{GeV}\right)^{-1} .
$$


Dividing Eq. (31) by the square of Eq. (32) gives

$$
M_{1} \simeq \frac{1}{a}\left(0.9 \times 10^{6} \mathrm{GeV}\right) .
$$

This is certainly small enough for satisfactory thermal leptogenesis. Dividing Eq. (31) by Eq. (32) yields

$$
\lambda \simeq \frac{1}{\left|f_{21}\right| a}\left(3 \times 10^{-5}\right),
$$

which indeed is of order $\eta / \epsilon=4 \times 10^{-5}$. For ease of writing, let us define $x \equiv f_{31} /\left|f_{21}\right|$ and $y \equiv u_{31} /\left|f_{21}\right|$. Then combining Eqs. (27) and (31) yields

$$
\tilde{m}_{1} \simeq \frac{1}{a}\left(3 \times 10^{-2} \mathrm{eV}\right)\left(1+|x|^{2}+|y|^{2}\right) .
$$

Assembling Eqs. (24), (25), (26), (34) and (35) (and using $(\ln k)^{3 / 5} \simeq 3$, which is a reasonable approximation for the values of $\tilde{m}_{1}$ that will be of interest) one has finally the result

$$
Y_{L} \simeq \frac{10^{-15}}{a}\left[\frac{\left(|x|^{2}-|y|^{2}\right) \operatorname{Im}\left(x^{*} y\right)}{\left(1+|x|^{2}+|y|^{2}\right)^{2}}\right] I .
$$

The maximum value of the function of $x$ and $y$ in the brackets is $1 / 4$. Thus sufficient leptogenesis requires a resonant enhancement factor of about $I \sim 4 \times 10^{5}$, well below the upper bound of $10^{10}$ imposed to ensure that the mass splitting of the quasi-Dirac pair is greater than their widths. If $a$ happens to be small, a smaller enhancement is needed, but for $a$ to be much less than one would be a fine-tuning.

\section{COMPARISON WITH LEPTOGENESIS IN TYPE I SEESAW}

To illustrate the advantages of the type III seesaw for both leptogenesis and obtaining realistic light neutrino masses, we shall briefly examine these issues in the realistic $S O(10)$ model that we have been using as a laboratory. Let us therefore now suppose that in that model only the conventional type I seesaw mechanism operates. It will be useful to parametrize the inverse of the matrix $M_{R}$ as follows:

$$
M_{R}^{-1}=\left[\begin{array}{ccc}
a\left(\frac{\epsilon}{\eta}\right)^{2} & b\left(\frac{1}{\eta}\right) & c\left(\frac{\epsilon}{\eta}\right) \\
b\left(\frac{1}{\eta}\right) & d\left(\frac{1}{\epsilon}\right)^{2} & e\left(\frac{1}{\epsilon}\right) \\
c\left(\frac{\epsilon}{\eta}\right) & e\left(\frac{1}{\epsilon}\right) & 1
\end{array}\right] m_{R}^{-1},
$$

where in order to get a realistic $M_{\nu}$, we take $a, b, c, d$, and $e$ to be of order one. Note that this matrix has a geometric hierarchy of the type that typically arises from an abelian flavor symmetry. From the form of $M_{N}$ given in Eq. (8) and the type I seesaw formula $M_{\nu}=-M_{N} M_{R}^{-1} M_{N}^{T}$, one has 


$$
M_{\nu}=-\left[\begin{array}{ccc}
a \epsilon^{2} & c \epsilon^{2} & (c-b) \epsilon \\
c \epsilon^{2} & \epsilon^{2} & (1-e) \epsilon \\
(c-b) \epsilon & (1-e) \epsilon & (1-2 e+d)
\end{array}\right] \frac{v_{u}^{2}}{m_{R}}
$$

The first thing to notice is that the ratio of the two larger light neutrino masses, $m_{2} / m_{3}$, is of order $\epsilon^{2}$. However, empirically this ratio is roughly equal to $\epsilon \cong 0.14$. Thus there must be a mild fine-tuning to make $(1-2 e+d)=O(\epsilon)$.

A much more precise tuning is required to have a large resonant enhancement of leptogenesis. Suppose that a resonant enhancement of $I \gg 1$ is required. Then the two lightest "right-handed" neutrinos, $N_{1}^{c}$ and $N_{2}^{c}$, must form a pseudo-Dirac pair with a mass splitting $\Delta M / M=1 /(2 I)$. Examining the 12 block of the matrix $M_{R}^{-1}$ in Eq. (37) one sees that this requires that $\left[d\left(\frac{1}{\epsilon}\right)^{2}+a\left(\frac{\epsilon}{\eta}\right)^{2}\right]=\frac{1}{2 I} b\left(\frac{1}{\eta}\right)$. That is, $a / b=\left[\eta /\left(2 \epsilon^{2}\right)\right] I^{-1}=1.5 \times 10^{-4} I^{-1}$.

A resonant enhancement of order $10^{4}$, then, would require a tuning of the parameter $a$ to be of order $10^{-8}$. This tuning of the 11 component of $M_{R}^{-1}$ is obviously equivalent to the tuning of the determinant of the 23 block of $M_{R}$. In Ref. [11] it is argued that in a particular version of this model (cf. [14]) this subdeterminant of $M_{R}$ may have a natural reason to be small compared to one, though not as small as $10^{-8}$.

We have considered a specific form of $M_{N}$ here that comes from a particular model of quark and lepton masses. However, this model is a fairly typical one, and it seems likely that a similar extreme fine-tuning of the form of $M_{R}$ would be required in a wide class of realistic models having the type I seesaw in order to produce large resonant enhancement of leptogenesis. This is the "clash" mentioned in the Introduction between the requirements of realistic $M_{\nu}$ and leptogenesis in type I seesaw models.

In the type III seesaw we found that a large resonant enhancement of leptogenesis required that the mass $M_{S}$ that sets the scale for the matrix $\mathcal{M}$ must be small compared to $M_{G U T}$. It would not be hard to make this natural by an abelian symmetry under which the singlets $S_{i}$ were charged. In any case, the setting of a mass scale such as $M_{S}$ to be small seems less unnatural than having a highly tuned relationship among the elements of the matrix $M_{R}$.

The research of SMB was supported in part by Department of Energy Grant Number DE FG02 91 ER 40626 A007. One of us (CHA) thanks the Fermilab Theoretical Physics Department for its kind hospitality where his work was carried out. Fermilab is operated by Universities Research Association Inc. under contract with the Department of Energy. 


\section{REFERENCES}

[1] M. Fukugita and T. Yanagida, Phys. Lett. B174, 45 (1986).

[2] M.A. Luty, Phys. Rev. D 45, 455 (1992); W. Buchmüller and T. Yanagida, Phys. Lett. B302, 240 (1993); H. Murayama and T. Yanagida, Phys. Lett. B322, 349 (1994); R. Jeannerot, Phys. Rev. Lett. 77, 3292 (1996).

[3] M. Flanz, E.A. Paschos, U. Sarkar, and J. Weiss, Phys. Lett. B389, 693 (1996); L. Covi and E. Roulet, Phys. Lett. B399, 113 (1997).

[4] W. Buchmüller and M. Plümacher, Phys. Lett. B431, 354 (1998); W. Buchmüller and T. Yanagida, Phys. Lett. B445, 399 (1999); M.S. Berger and B. Brahmachari, Phys. Rev. D 60, 073009 (1999); T. Asaka, K. Hamaguchi, M. Kawasaki, and T. Yanagida, Phys. Rev. D 61, 083512 (2000); J.R. Ellis, M. Raidel, T. Yanagida, Phys. Lett. B546, 228 (2002); G.C. Branco, R. Gonzalez Felipe, F.R. Joaquim, I. Masina, and C.A. Savoy, Phys. Rev. D 67, 073025 (2003); S. Pascoli, S.T. Petcov, and W. Rodejohann, Phys. Rev. D 68, 093007 (2003); W. Buchmüller, P. Di Bari, and M. Plümacher, Nucl. Phys. B665, 445 (2003); E. Akhmedov, M. Frigerio, and A.Yu. Smirnov, JHEP 0309, 021 (2003); A. Pilaftsis and T.E.J. Underwood, hep-ph/0309342.

[5] M. Gell-Mann, P. Ramond, and R. Slansky, in Supergravity, Proceedings of the Workshop. Stony Brook, New York, 1979, ed. P. van Nieuwenhuizen and D.Z. Freedman (North-Holland, Amsterdam, 1979), p.315; T. Yanagida, in Proc. Workshop on Unified Theory and the Baryon Number of the Universe, Tsukuba, Japan, 1979, ed. O. Sawada and A. Sugramoto (KEK Report No. 79-18, Tsukuba, 1979), p.95; R.N. Mohapatra and G. Senjanovic, Phys. Rev. Lett. 44, 912 (1980); S.L. Glashow, in Quarks and Leptons, Cargese (July 9-29, 1979), ed. M. Levy et al. (Plenum, New York, 1980), p.707.

[6] G. Lazarides, Q. Shafi, and C. Wetterich, Nucl. Phys. B181, 287 (1981), R.N. Mohapatra and G. Senjanovic, Phys. Rev. D 23, 165 (1981).

[7] T. Hambye and G. Senjanovic, hep-ph/0307237. See also E. Ma and U. Sarkar, Phys. Rev. Lett. 80, 5716 (1998); G. Lazarides and Q. Shafi, Phys. Rev. D 58, 071702 (1998).

[8] S.M. Barr, hep-ph/0309152.

[9] C.H. Albright and S.M. Barr, Phys. Lett. B452, 287 (1999); C.H. Albright, K.S. Babu, and S.M. Barr, Phys. Rev. Lett. 81, 1167 (1998).

[10] A. Pilaftsis, Phys. Rev. D 56, 5431 (1997); E. Akhmedov, M. Frigerio, and A.Yu. Smirnov, JHEP 0309, 021 (2003).

[11] C.H. Albright and S.M. Barr, in preparation.

[12] A. Pilaftsis, Phys. Rev. D 56, 5431 (1997).

[13] E.W. Kolb and M.S. Turner, The Early Universe, (Addison-Wesley, 1990); A. Pilaftsis, Int. J. Mod. Phys. A14, 1811 (1999); M. Flanz and E.A. Paschos, Phys. Rev. D 58, 113009 (1998).

[14] C.H. Albright and S.M. Barr, Phys. Rev. D 64, 073010 (2001). 


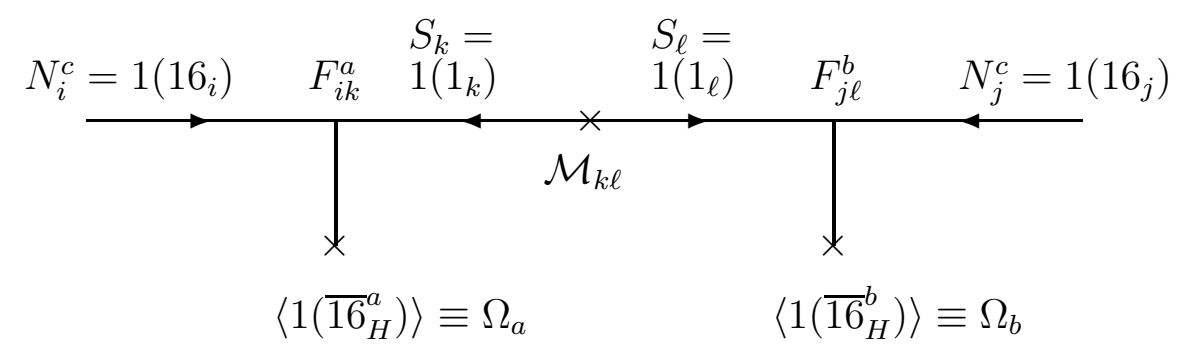

Fig. 1. Diagram that produces the effective operator $\mathbf{1 6}_{i} \mathbf{1 6}_{j} \overline{\mathbf{1 6}}_{H}^{a} \overline{\mathbf{1 6}}_{H}^{b} / M_{G}$, which generates $M_{R}$. 